\title{
IgA Vasculitis Complicated by Cytomegalovirus Enteritis: A Case Report
}

\author{
Risa Ninomiya, Tokuya Omi, Tokue Kato, \\ Nobuko Mayumi, Mai Kawarasaki and Seiji Kawana
}

Department of Dermatology, Nippon Medical School

\begin{abstract}
A 61-year-old man was admitted to our department with purpura and hemorrhagic bullae on his lower limbs, dull pain affecting the entire abdomen, and hematochezia. Histopathological examination and immunostaining revealed leukocytoclastic vasculitis of the small blood vessels of the dermis and IgA deposition; multiple ulcers were observed in the ileum during lower gastrointestinal (GI) endoscopy, so we made a diagnosis of IgA vasculitis (Henoch-Schönlein). Treatment with oral prednisolone (PSL) at a dose of $80 \mathrm{mg} / \mathrm{day}(1 \mathrm{mg} / \mathrm{kg} /$ day $)$ for one week resolved the symptoms almost completely. However, when the PSL dose was later reduced, dull epigastric pain and discomfort flared up again. Multiple punched-out ulcers were observed in the duodenum during upper GI endoscopy, and immunostaining revealed cytomegalovirus (CMV) in vascular endothelial cells and infiltrating cells. The patient's serum was positive for CMV antigenemia. On the basis of these findings, we concluded that the CMV enteritis had developed as a complication arising from the patient's immunosuppressed state, which was itself a result of the steroid therapy. We treated the patient with ganciclovir, which relieved the abdominal symptoms.
\end{abstract}

(J Nippon Med Sch 2014; 81: 48-52)

Key words: $\operatorname{Ig}$ A vasculitis, cytomegalovirus enteritis, corticosteroid therapy, immunosuppression

\section{Introduction}

In recent years, the number of cases of cytomegalovirus (CMV) enteritis has increased among patients who are immunocompromised because of such factors as acquired immunodeficiency syndrome (AIDS), organ transplantation, and chemotherapy; the problem is also seen in patients with systemic connective tissue diseases treated with long-term steroid therapy, including rheumatoid arthritis, collagen diseases, and vasculitis ${ }^{1}$.

The main symptoms of CMV enteritis are abdominal pain and diarrhea. When small bowel perforation or necrosis develops, a laparotomy is needed, and some cases of death have been reported $^{1-5}$.

We treated a patient with CMV enteritis as a complication of IgA vasculitis treated with a highdose steroid. Because the CMV enteritis was diagnosed early and treated with ganciclovir, it was cured relatively easily.

Correspondence to Seiji Kawana, MD, PhD, Department of Dermatology, Nippon Medical School, 1-1-5 Sendagi, Bunkyo-ku, Tokyo 113-8603, Japan

E-mail: kawa2559@nms.ac.jp

Journal Website (http://www.nms.ac.jp/jnms/) 

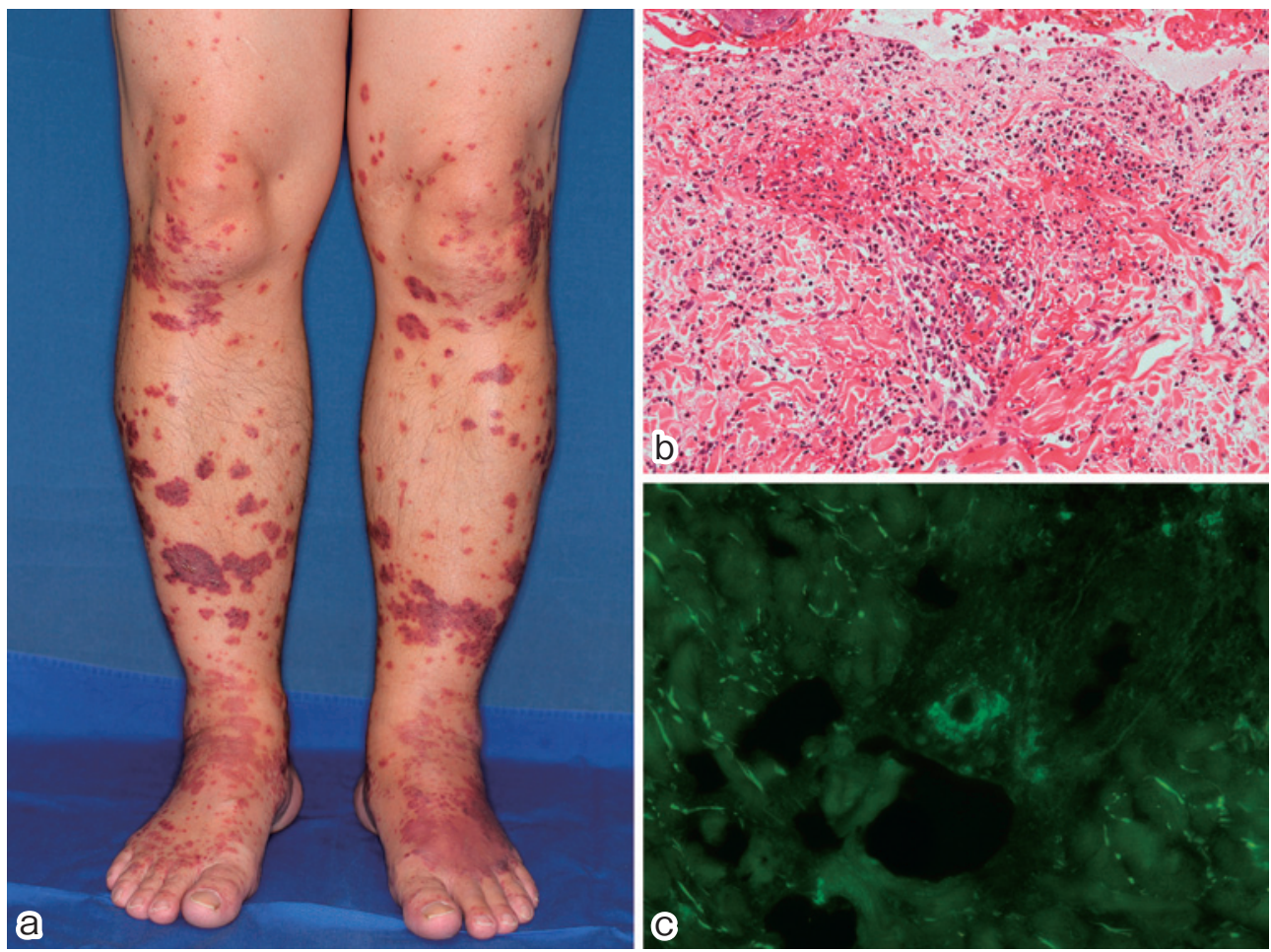

Fig. 1. a: Skin lesions on the lower extremities: multiple palpable purpura and hemorrhagic bullae measuring from $2 \mathrm{~mm}$ to $2 \mathrm{~cm}$ in diameter were observed on both legs.

b: Histopathological findings in the skin: leukocytoclastic vasculitis of the small blood vessels in the dermis was observed. Subepidermal blisters filled with neutrophils and fibrin were seen inside.

c: Direct immunofluorescence: granular $\operatorname{IgA}$ deposits were seen in the walls of the small blood vessels in the dermis.

\section{Case Report}

A 61-year-old man was admitted to our department with a 10-day history of knee and ankle pain and swelling, purpura covering the lower limbs, dull pain affecting the entire abdomen combined with a sensation of abdominal bloating, and hematochezia. Initial examination revealed multiple areas of palpable purpura and hemorrhagic bullae measuring $2 \mathrm{~mm}$ to $2 \mathrm{~cm}$ in diameter on the patient's limbs, buttocks, and abdomen (Fig. 1a).

Clinical laboratory tests revealed peripheral leukocytosis and an elevated C-reactive protein (CRP) value. A urine dipstick test was positive for $+/-$ of blood and $1+$ of protein. The fecal hemoglobin level was $12,788 \mathrm{ng} / \mathrm{mL}$. Blood coagulation factor XIII activity was 38\% (normal range: 70-140\%), a level that correlates with abdominal symptoms ${ }^{6}$.
Histopathological examination of the purpura and hemorrhagic bullae on the legs revealed leukocytoclastic vasculitis of the small blood vessels in the upper and middle layers of the dermis, and subepidermal blisters filled with fibrin and neutrophils. Direct immunofluorescence assay showed granular deposition of IgA and C3 in the walls of the small blood vessels of the dermis (Fig. 1 b, c).

Upper gastrointestinal (GI) endoscopy showed patchy redness and erosions of the gastric mucosa and duodenal mucosa. Lower GI endoscopy revealed mucosal edema, erosions, and deep ulcers with irregular margins at multiple sites in the ileum (Fig. 2a). Histopathological examination of the deep ulcers revealed intense neutrophil infiltration and bleeding in the lamina propria mucosae, and direct immunofluorescence assay demonstrated IgA deposition in the walls of the small blood vessels (Fig. 2b, c). 

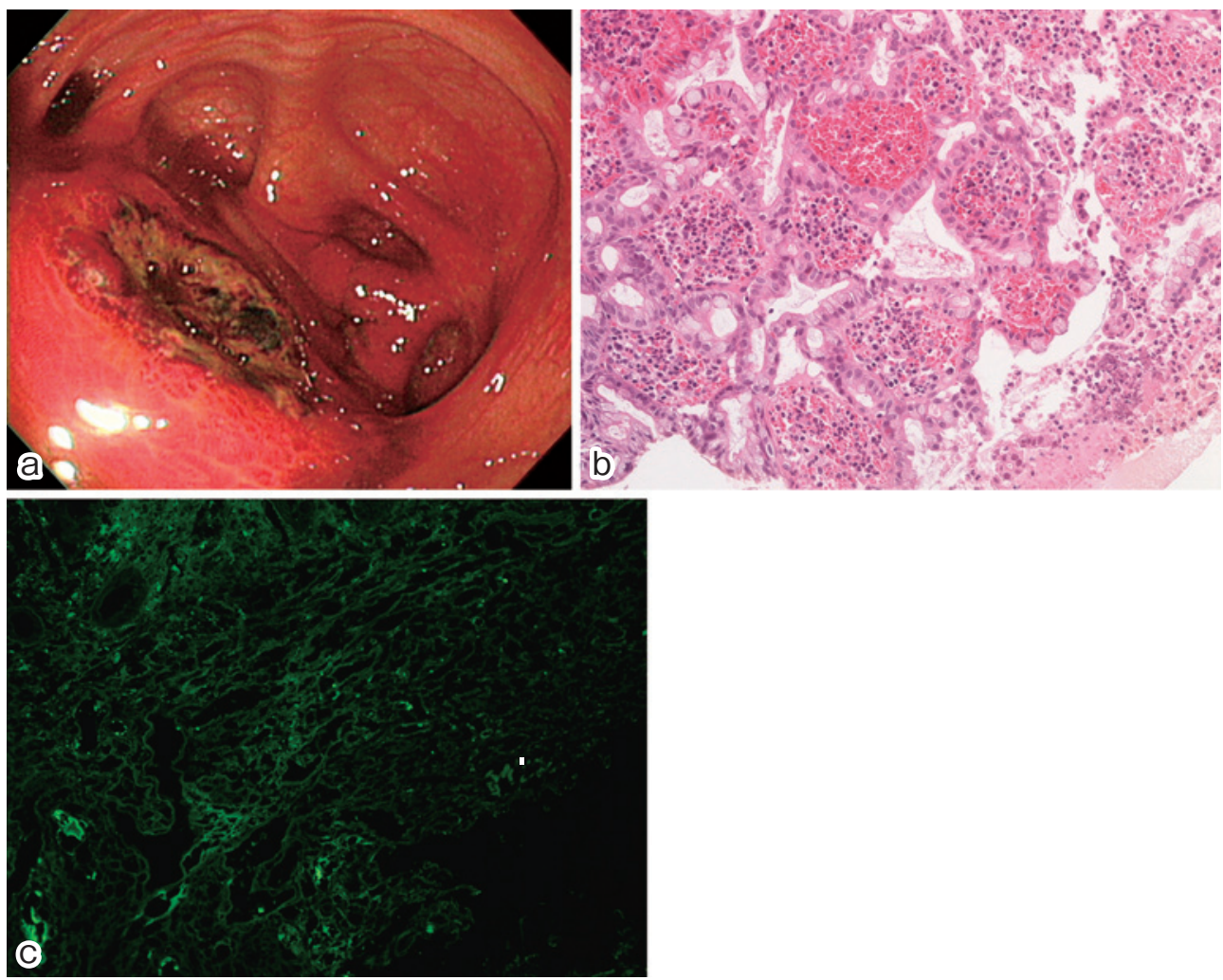

Fig. 2. a: Lower intestinal endoscopy revealed ulcers with irregular margins accompanied by bleeding and necrosis.

b: Histopathological examination of the ulcer showed neutrophil infiltration and hemorrhages intermingled with nuclear dust in the lamina propria mucosae.

c: A direct immunofluorescent study revealed deposition of $\operatorname{Ig} \mathrm{A}$ in the walls of the small blood vessels of the lamina propria mucosae.

On the basis of the above findings we diagnosed IgA vasculitis, which we treated with intravenous prednisolone (PSL) $60 \mathrm{mg} /$ day $(0.75 \mathrm{mg} / \mathrm{kg} /$ day $)$ for 7 days. But because the abdominal pain and hematochezia persisted, we switched to oral PSL and increased the dose to $80 \mathrm{mg} /$ day $(1 \mathrm{mg} / \mathrm{kg} /$ day $)$. One week later, the purpura and abdominal pain had subsided, but when we reduced the dose of PSL to $60 \mathrm{mg} /$ day, epigastric pain and discomfort flared up again. Upper GI endoscopy showed that shallow ulcers with regular margins had developed in the duodenum (Fig. 3a), leading us to suspect CMV enteritis. In addition, the serum CMV IgM titer had risen to 1.41 , and the patient's serum was positive for $\mathrm{CMV}$ antigenemia. Immunostaining revealed CMV inclusion bodies that stained positively with a mouse anti-CMV monoclonal antibody (Code No: M0854, DAKO, Denmark) in the nuclei of the vascular endothelial cells and infiltrating cells in the duodenal mucosa (Fig. 3b). Lower GI endoscopy, on the other hand, showed that the ulcers discovered in the ileum during the previous examination had healed and scarred. We concluded, therefore, that the abdominal symptoms that had flared up were not due to progression of the $\operatorname{Ig} \mathrm{A}$ vasculitis, but due to CMV enteritis. We started treatment with ganciclovir $(2.5 \mathrm{mg} / \mathrm{kg})$, and 10 days later the abdominal symptoms had improved and tests for CMV antigenemia were negative. Because occult hematuria and proteinuria later increased, as did serum creatinine levels, we added mizoribine (150 $\mathrm{mg}$ /day) to the treatment regimen. One month later, the proteinuria and occult hematuria had both been resolved, and the patient was discharged. We have continued treatment with PSL $(2.5 \mathrm{mg} /$ day $)$ on alternate days and mizoribine $(100 \mathrm{mg} /$ day), and as of one year later, there have been no recurrences of the abdominal symptoms or renal manifestations. 

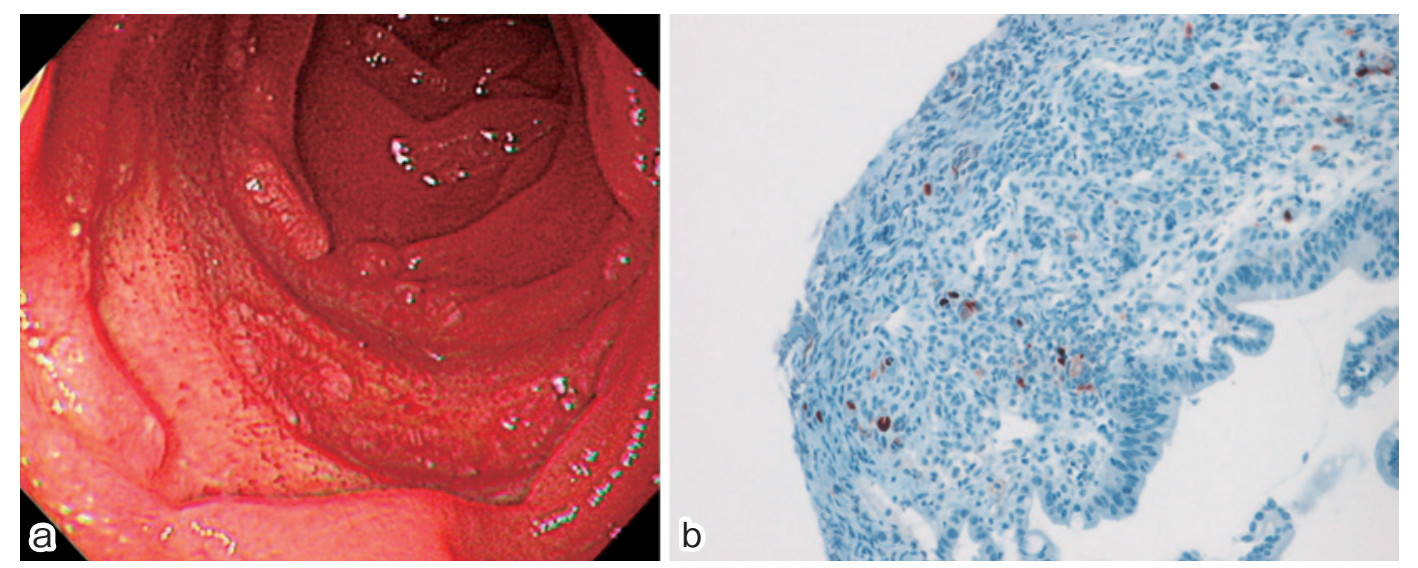

Fig. 3. a: Upper GI endoscopy revealed shallow ulcers with regular margins in the duodenum.

b: Histopathological examination of the ulcer: immunostaining with a mouse anticytomegalovirus monoclonal antibody showed that the nuclei and cytoplasm were positive for CMV inclusions bodies.

\section{Discussion}

GI manifestations of IgA vasculitis, which include upper abdominal pain, nausea and vomiting, diarrhea, and hematochezia, are seen in $56 \%$ to $85 \%$ of adults ${ }^{7}$ PSL at a dose of $1 \mathrm{mg} / \mathrm{kg} /$ day is effective in reducing severe GI symptoms and is recommended as the drug of first choice ${ }^{8}$. We treated the patient described herein with PSL at an initial dose of $60-80 \mathrm{mg} /$ day $(0.75-1 \mathrm{mg} / \mathrm{kg} /$ day), and the total steroid dose before CMV enteritis developed as a complication was $1,710 \mathrm{mg}$.

The CMV antibody prevalence rate in adults is $40 \%-90 \%$, and once they are infected, the virus remains dormant in the body and reactivates if the host's immunity declines. CMV can cause encephalitis, enteritis, and interstitial pneumonitis in adults with immune deficiency, as seen, for example, in patients with AIDS, those undergoing organ transplantation or chemotherapy, and patients on long-term steroid therapy ${ }^{1}$.

It has been reported that three diagnostic criteria must be met for a diagnosis of CMV infection to be made: (1) the presence of clinical manifestations, (2) the presence of organ lesions, and (3) demonstration of $\mathrm{CMV}$ in the affected tissue ${ }^{9}$. In the case of enteritis, the three diagnostic criteria are: (1) GI manifestations (diarrhea, hematochezia, abdominal pain, etc.), (2) GI ulcers and erosion, and (3) demonstration of $\mathrm{CMV}$ in the tissue. A positive result for serum CMV antigenemia can be substituted for (3).

Abdominal pain, hematochezia, and diarrhea are GI manifestations of both CMV enteritis and IgA vasculitis, and it is impossible to distinguish between the two conditions on the basis of these symptoms alone. When our patient's abdominal pain flared up in the form of epigastric pain after once improving, we thought at first that it was a GI symptom of $\operatorname{Ig}$ A vasculitis. However, an endoscopic examination showed that the earlier lesions of the ileal mucosa had healed and that punched-out ulcers had newly developed in the duodenum. Histological examination of the ulcer revealed CMV inclusion bodies, and the serum was positive for CMV antigenemia. Therefore, we made a definite diagnosis of CMV enteritis.

As far as we were able to determine in a search of the literature, only 2 cases of $\operatorname{IgA}$ vasculitis complicated by CMV enteritis have ever been reported $^{4,10}$. In both patients, abdominal pain and hematochezia occurred during steroid therapy. One of the patients had a small bowel perforation, which was successfully treated with an enterectomy, but the other patient died of hemorrhagic shock. Steroids are ineffective against CMV enteritis, and it is usually treated with intravenous ganciclovir administration $(5 \mathrm{mg} / \mathrm{kg} /$ day divided into 2 doses a day) for 7-14 days. But when CMV enteritis leads to 
perforation, bleeding, or necrosis, a laparotomy is needed, and in some cases patients have died because of the bleeding ${ }^{2-5}$. In our patient, early diagnosis meant that we were able to treat the CMV enteritis successfully and prevent progression to bowel necrosis and perforation.

When severe abdominal symptoms flare up or linger in a patient on steroid therapy for $\operatorname{Ig} \mathrm{A}$ vasculitis, the possibility of complication with $\mathrm{CMV}$ enteritis should be considered; simply increasing the steroid dose can worsen the patient's condition and even lead to death.

Conflict of Interest: The authors declare no conflict of interest

\section{References}

1. Goodgame RW: Gastrointestinal cytomegalovirus disease. Ann Intern Med 1993; 119: 924-935.

2. Bang S, Park YB, Kang BS, et al.: CMV enteritis causing ileal perforation in underlying lupus enteritis. Clin Rheumatol 2004; 23: 69-72.

3. Hara M, Tomoda F, Nakagawa T, et al.: An autopsy case of Henoch-Schönlein purpura nephritis complicated with intestinal cytomegalovirus infection. Nihon Jinzo Gakkai Shi 2010; 52: 1037-1042
[Abstract in English].

4. Strasser C, Wolf EM, Komprat P, et al:: Opportunistic cytomegalovirus infection causing colonic perforation in a patient with systemic lupus erythematosus. Lupus 2012; 21: 449-451.

5. Ishiguro $\mathrm{T}$, Takayanagi $\mathrm{T}$, Kawabata $\mathrm{Y}$, et al: Intestinal perforation due to concomitant cytomegalovirus infection during treatment for Pneumocystis jirovecii pneumonia in a patient with rheumatoid arthritis. Intern Med 2011; 50: 1835-1837.

6. Matayoshi T, Omi T, Sakai N, Kawana S: Clinical Significance of Blood Coagulation Factor XIII Activity in Adult Henoch-Schönlein Purpura. J Nippon Med Sch 2013; 80: 268-278.

7. Esaki M, Matsumoto T, Nakamura S, et al.: GI involvement in Henoch-Schönlein purpura Gastrointest Endosc 2002; 56: 920-923.

8. Ronkainen J, Koskimies O, Ala Houhala M, et al: Early prednisolone therapy on Henoch-Schonlein purpura: a randomised, double-blind placebocontrolled trial. J Pediatr 2006; 149: 241-247.

9. Ljungman P, Plotkin SA: Workshop on CMV disease; definitions, clinical severity scores, and new syndromes. Scand J Infect Dis 1995; Suppl (99): 87.

10. Nguyen-Ho P, Jewell LD, Thomson AB: Hemorrhagic intestinal Henoch-Schonlein purpura complicated by cytomegalovirus infection. Can J Gastroenterol 1998; 12: $71-77$.

(Received, September 20, 2013)

(Accepted, October 11, 2013) 